\title{
Lanthanide Europium MOF Nanocomposite as the Theranostic Nanoplatform for Microwave Thermo- Chemotherapy and Fluorescence Imaging
}

\section{Lirong Zhao}

Technical Institute of Physics and Chemistry CAS: Technical Institute of Physics and Chemistry

\section{Wei Zhang}

Jinan University First Clinical Hospital: Shenzhen People's Hospital

\section{Qiong Wu}

Technical Institute of Physics and Chemistry CAS: Technical Institute of Physics and Chemistry

\section{Changhui Fu}

Technical Institute of Physics and Chemistry CAS: Technical Institute of Physics and Chemistry

\section{Xiangling Ren}

Technical Institute of Physics and Chemistry CAS: Technical Institute of Physics and Chemistry

\section{Kongpeng LV}

Jinan University First Clinical Hospital: Shenzhen People's Hospital

\section{Tengchuang Ma}

Harbin Medical University

\section{Xudong Chen}

Jinan University First Clinical Hospital: Shenzhen People's Hospital

\section{Longfei Tan}

Technical Institute of Physics and Chemistry CAS: Technical Institute of Physics and Chemistry Xianwei Meng ( $\nabla$ mengxw@mail.ipc.ac.cn)

Technical Institute of Physics and Chemistry CAS: Technical Institute of Physics and Chemistry https://orcid.org/0000-0002-0350-4760

\section{Research Article}

Keywords: Lanthanide metal organic frameworks, EuMOF, Microwave thermal therapy, Fluorescence imaging, Theranostic nanoplatform

Posted Date: December 20th, 2021

DOI: https://doi.org/10.21203/rs.3.rs-1173765/v1 
License: (c) (i) This work is licensed under a Creative Commons Attribution 4.0 International License. Read Full License

Version of Record: A version of this preprint was published at Journal of Nanobiotechnology on March 15th, 2022. See the published version at https://doi.org/10.1186/s12951-022-01335-7. 


\section{Abstract \\ Backgrounds:}

Microwave sensitization nanoplatform, integrating multiple functional units for improving tumor selectivity, is of great significance for clinical tumor microwave therapy. Lanthanide europium metal organic framework materials (EuMOF) are expected to be a theranostic nanoplatform owing to its specific luminescent properties and microwave sensitization properties. However, it is difficult to be applied to complex biological systems for EuMOF due to its rapid degradation induced by the solvent molecular and ionic environment. In this work, a luminescent EuMOF nanocomposite (EuMOF@ZIF/APPEG, named EZAP) was designed, which brought the multifunctional characteristics of microwave sensitization, fluorescence imaging and drug loading.

\section{Results}

Lamellar EuMOF was synthesized by a hydrothermal method. Through the charge adsorption mechanism, the zeolite imidazole framework (ZIF) structure was densely assembled on the surface of EuMOF to realize the protection. Then, through in-situ apatinib drug loading and PEG modification, EZAP nanocomposite was finally obtained. Apatinib (AP) was a kind of chemotherapy drug approved by Food and Drug Administration for clinical use. PEG modification increased long-term circulation of EZAP nanocomposite. The physical and chemical structure and properties of EuMOF@ZIF (EZ) were systematically represented, indicating the successful synthesis of the nanocomposite. The toxic and side effects were negligible at a safe dose. The growth of human liver cancer cells and murine liver cancer cells in vitro was significantly inhibited, and the combined microwave-thermal therapy and chemotherapy in vivo achieved high anti-cancer efficacy. Moreover, EZAP nanocomposite possessed bright red fluorescence, which had good ability for tumor imaging in tumor-bearing mice in vivo.

\section{Conclusion}

Therefore, EZAP nanocomposite showed high microwave sensitization, excellent fluorescence properties and good drug loading capacity, establishing a promising theranostic nanoplatform for tumor therapy and fluorescence imaging. This work proposes a unique strategy to design for the first time a multifunctional nanoplatform with lanthanide metal organic frameworks for tumor treatment and diagnosis in the biological application.

\section{Introduction}

Microwave thermal therapy, as a new type of localized cancer treatment for tumor thermal ablation, takes the advantages of minimally invasion and deep penetration [1,2]. Microwave thermal therapy has been widely used in the clinical treatment of tumors, and is currently the first-line treatment for hepatocellular 
carcinoma [3-7]. However, microwave thermal therapy is not selective for tumor cells and does not specifically damage to them [8-10]. Thus tumor cells are hardly ablated completely, there will be a high recurrence rate. It also results obvious cytotoxic effects on surrounding healthy tissues. Hence, a variety of microwave sensitizing nanomaterials have been developed [11-14], such as organic materials (gelatin, sodium alginate microcapsules) [15], inorganic materials [16-18] (zirconium dioxide hollow nanospheres [19], carbon nanotubes [20-22]), organic-inorganic hybrid materials (metal organic framework [23-25]). Amongst, synthesis methods and the targeting ability of the organic materials need to be improved [26, 27]. The inorganic nanomaterials are unable to be metabolized and difficult to be eliminated from the body, which limits their clinical applications [28-30]. Metal organic framework materials, as organic and inorganic hybrid materials, are widely used in tumor treatment due to their low toxicity and good biodegradability [31-33]. Most recently, the MOFs become an optional microwave sensitization agents due to the effective electromagnetic response leading from the strong scattering, reflection and dissipation by their interconnected network structure with high porosity and large specific surface area [34].

As a luminescent material, lanthanide metal organic frameworks (LnMOFs) have shown attractive application prospects in the fields of chemical sensing, temperature sensing, biological detection and biological imaging $[35,36]$. Of particular note, abundant electron energy levels and a large number of energy level transitions in the electron configuration endow the lanthanide europium metal organic frameworks with the most potential microwave sensitization agents [37-39]. The additional vital part of Eu metal organic framework is the specific fluorescence, high quantum efficiency, long fluorescence lifetime and characteristic emission peak position, as imaging agent plays the important role for disease diagnosis and treatment. Therefore, EuMOF is a promising candidate for efficacy theranostic agent to integrating the high microwave sensitizing and fluorescent imaging [40,41].

However, the research of EuMOF in nanomedicine field has hardly been carried out and reported, especially for combined treatment of tumors. Because EuMOF was easily degraded by the surrounding ionic environment and solvent molecules, resulting in fluorescence quenching [42]. Until now, the EuMOF theranostic nanoplatform has not been achieved for that [43].

Here, to solve this problem, a stable zeolite imidazole framework structure was introduced on the surface of Eu MOF to provide coating protection for EuMOF, while as an excellent drug loading tool due to its porosity and high specific surface [44-46]. Therefore, we reported a luminescent EuMOF nanocomposite (EuMOF@ZIF/AP-PEG, named EZAP) as a multifunctional nanoplatform for microwave-thermal therapy and chemotherapy and fluorescence imaging of tumors (Scheme 1). EuMOF was synthesized by solvothermal method. After ZIF growth on the surface of EuMOF, in-situ apatinib loading and PEG modification, EZAP nanocomposite was finally obtained [47]. EZAP nanocomposite showed high microwave sensitization, excellent fluorescence properties and good drug loading capacity. The physical and chemical structure and properties of EZ, drug loading in vitro and tumor fluorescence imaging in vivo and in vitro were systematically studied. The anti-tumor effect of EZAP nanocomposite under microwave irradiation in vivo and in vitro was studied in detail. The tumor fluorescence imaging was evaluated to 
prove the potential of EuMOF@ZIF as a fluorescent nanoprobe. In this multifunctional platform, we not only created a new-type microwave sensitization material, but also innovatively pushed the lanthanide MOF to the living level in the field of microwave-responsive nanomedicine, including in vivo tumor imaging and treatment. It also provides a novel idea for the biological application of lanthanide MOFs.

\section{Methods}

\section{Materials}

Europium nitrate hexahydrate, 1, 4-naphthalene dicarboxylic acid, cetyl trimethyl ammonium bromide, zinc nitrate hexahydrate, 2-methylimidazole, triethanolamine, polyvinylpyrrolidone, n, ndimethylformamide, absolute ethanol, absolute methanol, all reagents are analytically pure.

\section{Synthesis of EuMOF}

Nano-scale EuMOF was synthesized using europium nitrate hexahydrate $\left(\mathrm{Eu}\left(\mathrm{NO}_{3}\right)_{3} \cdot 6 \mathrm{H}_{2} \mathrm{O}\right)$ and 1, 4naphthalene dicarboxylic acid $(1,4-\mathrm{H} 2 \mathrm{NDC})$ as raw materials. The metal nitrate $\mathrm{Eu}\left(\mathrm{NO}_{3}\right)_{3} \cdot 6 \mathrm{H}_{2} \mathrm{O}(1.25$ $\mathrm{mmol})$ and the organic ligand 1,4-H2NDC $(1.25 \mathrm{mmol})$ were added into the solvent DMF $(100 \mathrm{~mL})$ in equal proportion. After that, surfactant CTAB and $\mathrm{pH}$ regulator TEOA(NCTAB:NTEOA=1:2) were added, as well as dispersant PVP (100 mg). After stirring and mixing evenly, the mixed solution is added to the appropriate size of the reaction kettle. After reaction for $2 \mathrm{~h}$ in the oven at $100{ }^{\circ} \mathrm{C}$, centrifugation at 10000 rpm for 5 min was performed successively with ethanol washing, water washing and ethanol washing. Freeze-drying produces a white product.

\section{Synthesis and characterization of EuMOF@ZIF}

Nanoscale EuMOF was synthesized using europium nitrate hexahydrate and 1, 4-naphthalene dicarboxylic acid as raw materials. EuMOF was dispersed in ethanol, and PVP was dissolved in ethanol. PVP alcohol solution was added drop by drop into EuMOF solution, stirred by magnetic force at room temperature for $4 \mathrm{~h}$, centrifuged at $10000 \mathrm{rpm}$ for $5 \mathrm{~min}$, washed with anhydrous ethanol for 3 times, and freeze-dried to obtain white product. Weighed $20 \mathrm{mg}$ of the above products and dispersed them in $10 \mathrm{~mL}$ methanol, added $1 \mathrm{~mL}$ of $\mathrm{Zn}\left(\mathrm{NO}_{3}\right)_{3} \cdot 6 \mathrm{H}_{2} \mathrm{O}(0.27 \mathrm{~mol} / \mathrm{mL})$ methanol solution, stirred for 10 min, added 3 $\mathrm{mL}$ of $2-\mathrm{Ml}(0.65 \mathrm{~mol} / \mathrm{mL})$ methanol solution, stirred at room temperature for $6 \mathrm{~h}$. The reaction was centrifuged at $8000 \mathrm{rpm}$ for $5 \mathrm{~min}$, washed with anhydrous ethanol for 3 times, and freeze-dried to obtain a white product the EuMOF@ZIF nanocomposite. Scanning electron microscopy (S-4800, Hitachi) and biological transmission electron microscopy (HT7700, Hitachi) were used to observe the morphology of the EuMOF@ZIF nanocomposite. The elemental distribution and content of the samples were determined by high resolution transmission electron microscopy (JEM-2100F, Hitachi). The hydrated particle size and potential of the sample were measured by the Particle size potential analyzer (Nano ZSE, Malvern). X-ray 
diffractive analysis (D8 focus, Bruker) of the sample was performed with CuKa light source at a scanning rate of $0.02 \mathrm{deg} / \mathrm{s}$. The infrared absorption curves of the samples were determined by the Fourier infrared absorption (Excalibur 3100, Varian) analyzer. The ultraviolet absorption curves of the samples were measured by UV-vis spectrophotometer (Cary5000, Agilent).

\section{In vitro microwave thermal performance}

The prepared EuMOF@ZIF nanocomposite was tested by microwave heating to study its microwave heating effect. Firstly, EuMOF@ZIF nanomaterials with a concentration gradient of 0, 2, 4, $8 \mathrm{mg} / \mathrm{mL}$ were prepared by using $0.9 \% \mathrm{NaCl}$ as the solvent. One $\mathrm{mL}$ of the samples was put into the sample tank of the microwave instrument, and the microwave irradiation was carried out at a frequency of $1.8 \mathrm{~W}$ for 5 min. The temperature data were recorded every $10 \mathrm{~s}$. During this process, the red-heat external imager was used to monitor the temperature changes in real time.

\section{In vitro luminescent properties}

The fluorescence test of the prepared EuMOF@ZIF nanocomposite proves its excellent properties. The best excitation wavelength of the nanomaterial is $280 \mathrm{~nm}$, and the maximum emission peak is $618 \mathrm{~nm}$. Firstly, under the condition of certain material concentration, the fluorescence curves of the EuMOF@ZIF nanocomposite at different excitation wavelengths were obtained, and then the emission spectra of materials with different concentrations $(5,4,2,1,0.5,0.25,0.125,0.0625,0.03125 \mathrm{mg} / \mathrm{mL})$ at the optimal excitation wavelengths were explored.

\section{In vitro drug loading and release behavior}

In order to study the treatment in animals, PEG modification was carried out to make it have long circulation characteristics. The EuMOF@ZIF nanocomposite was weighed $10 \mathrm{mg}$ and dispersed in $3 \mathrm{~mL}$ ethanol, and the same volume of $\mathrm{SH}_{2}-\mathrm{PEG}(10 \mathrm{mg})$ alcohol solution was added. The magnetic force was stirred at room temperature for $2 \mathrm{~h}$. Centrifuged at 8,000 rpm for $5 \mathrm{~min}$, washed with anhydrous ethanol for 3 times, freeze-dried to obtain white product EuMOF@ZIF-PEG nanocomposite for later use. The preparation of EuMOF@ZIF/AP-PEG nanocomposite is equivalent to the drug loading process, and the material is prepared by in-situ loading with a mass ratio of 1.0 to apatinib. The specific operations are as follows: EuMOF@ZIF nanocomposite $10 \mathrm{mg}$ were dispersed in $3 \mathrm{~mL}$ ethanol to prepare $1 \mathrm{~mL}$ of apatinib solution $(10 \mathrm{mg} / \mathrm{mL})$. The two were mixed and stirred evenly, then $1 \mathrm{~mL}$ of capto polyethylene glycol solution $(10 \mathrm{mg} / \mathrm{mL})$ was added and stirred at room temperature for $4 \mathrm{~h}$. The solution was centrifuged and washed three times with ethanol. The EuMOF@ZIF/AP-PEG nanocomposite containing chemotherapeutic drugs was obtained. The supernatant was collected and diluted to an appropriate volume at a certain multiple. The UV absorption of Apatinib was detected and the loading rate was calculated. The standard concentration-absorption curve of apatinib was Abs $=0.02057 \mathrm{C}+0.00659$ 
$\left(R^{2}=0.9999\right)$ according to our group's previous report. The precipitates were modified with PEG and drug release experiments were carried out under neutral physiological conditions. The standard curve of drug release was drawn, and the amount of drug release was calculated according to the absorbance value of drug release.

\section{Cytotoxicity}

The biosafety of EZ nanocomposite was evaluated after PEG modification. HepG2 cells were incubated with $5 \% \mathrm{CO}_{2}$ in the incubator at $37^{\circ} \mathrm{C}$. When the density of HepG2 cells grew to $70-80 \%$, the old medium was sucked out, washed by PBS for 3 times, and then trypsin was added to digest HepG2 cells. The trypsin was sucked out, the newly prepared medium was added, the digested cells were gently blown and placed in a 96-well plate for $24 \mathrm{~h}$. Then different concentrations $(0,12.5,25,50,100,200 \mu \mathrm{g} / \mathrm{mL})$ of the EuMOF@ZIF-PEG nanocomposite was co-cultured with cells for $24 \mathrm{~h}$, and then MTT $(0.5 \mathrm{mg} / \mathrm{mL})$ was added into the culture system. After the MTT was removed, dimethyl sulfoxide was added. The absorbance (absorption peak $492 \mathrm{~nm}$ ) of each plate on the 96 -well plate was measured using a microplate analyzer to calculate the cell viability and evaluate the biosafety.

\section{VEGF immunofluorescence test}

HepG2 cells were used as cell model to evaluate the inhibitory effect of EuMOF@ZIF/AP-PEG on vascular endothelial growth factor expression. Tumor cells were cultured at $37{ }^{\circ} \mathrm{C}, 5 \% \mathrm{CO}_{2}$. The experiment was divided into four groups (control, Apatinib, EZAP, EZP). After $24 \mathrm{~h}$ co-incubation with cells in each group, the fluorescence of cells was observed under a fluorescence microscope after fixation, washing, sealing, primary antibody incubation, washing, secondary antibody incubation with green fluorescence, washing, DAPI staining with blue fluorescence and other steps.

\section{In vitro microwave thermo-chemotherapy evaluation}

To evaluate the microwave thermal-chemotherapy performance of composite nanomaterials at the cell level, tumor cell inhibition experiments were carried out. HepG2 cells were evenly seeded into 6-well plates and cultured until cells adhered to the wall. The cells were divided into seven groups and treated with different treatment methods :1) control group, 2) free Apatinib group, 3) EuMOF@ZIF-PEG group, 4) EuMOF@ZIF/AP-PEG group, 5) MW group, 6) EuMOF@ZIF-PEG + MW group, 7) EuMOF@ZIF/AP-PEG + MW group. The details are as follows: new medium was added to the groups 1 and 5; equivalent medium (Apatinib concentration was $20 \mu \mathrm{g} / \mathrm{mL}$ ) was added in the group 2. The group 3 and group 6 were added with equivalent medium (EuMOF@ZIF-PEG concentration was $100 \mu \mathrm{g} / \mathrm{mL}$ ); Groups 4 and group 7 were added with equivalent culture medium (EuMOF@ZIF/AP-PEG concentration was $100 \mu \mathrm{g} / \mathrm{mL}$ ) for $24 \mathrm{~h}$, then the cells were digested with trypsin, centrifuged, the culture medium was removed, $1 \mathrm{~mL}$ of culture medium was added, and the cells of each group were dispersed evenly by beating. Free Apatinib group, 
EuMOF@ZIF-PEG group, EuMOF@ZIF/AP-PEG group were transferred to 96-well plate at $100 \mu \mathrm{L} /$ well. The other three groups were MW group, EuMOF@ZIF-PEG + MW group, EuMOF@ZIF/AP-PEG + MW group were digested, centrifuged, and the medium was removed. DMEM was gently blown and dispersed, irradiated with MW (1.8 W) for 5 min, centrifuged, DMEM was poured, $1 \mathrm{~mL}$ medium was added, and then added into 96 -well plate at $100 \mu \mathrm{L}$ per well for $24 \mathrm{~h}$. MTT assay was performed to calculate cell viability and evaluate antitumor efficiency.

In order to further intuitively verify the tumor inhibition effect under microwave irradiation, living-dead cell staining experiment was carried out. Like that, after each group of cells had been treated, they were inoculated into a six-well plate. Adding green fluorescent calcium xanthocyanin solution and red fluorescent propidium iodide solution under dark condition, respectively, gently shaking, avoid light, a total of 10 min incubation in the tumor cells. Then, the cell staining was observed under a fluorescent confocal microscope to obtain the cell death status and evaluate the inhibition effect of tumor cells.

\section{In vivo microwave thermo-chemotherapy}

Female BALB/c mice $\ 16-18 \mathrm{~g} \Downarrow$ were used as animal model. After hair removal, H22 tumor cells $\nabla 100 \mu \mathrm{L} \bigotimes$ $2 \times 10^{6}$ \were inoculated into the armpits of mice. When the tumor grows to the right size $\left(130 \pm 30 \mathrm{~mm}^{3}\right)$, tumor-bearing mice were randomly divided into six groups with three mice in each group: control group (without any treatment), EuMOF@ZIF/AP-PEG group (containing drug material, tail i.v., 50 mg/kg), free Apatinib group (tail i.v.,10 mg/kg), MW group (1.8 W, 5 min), EuMOF@ZIF-PEG + MW group (without drug material plus microwave, tail i.v., 50 mg/kg, 1.8 W, 5 min), EuMOF@ZIF/AP-PEG + MW group (drug containing materials plus microwave, tail i.v., $50 \mathrm{mg} / \mathrm{kg}, 1.8 \mathrm{~W}, 5 \mathrm{~min}$ ). During this period, the temperature changes of mice tumors during microwave treatment were monitored in real time by using the infrared thermal imager. Daily changes in body weight, tumor volume and survival of each mouse in each group were recorded, and photos were taken for preservation. Mouse tumor volume was calculated according to the following formula: Mouse tumor volume $=\left(\right.$ length * width $\left.{ }^{2}\right) / 2$, the unit was $\mathrm{mm}^{3}$. After 14 consecutive days of observation, curves were drawn according to the tumor volume of mice to evaluate the therapeutic effect of EuMOF@ZIF/AP-PEG nanocomposite on liver cancer in vivo.

\section{In vivo fluorescence imaging}

In addition, fluorescence imaging signals of tumor-bearing mice were collected at different time points using the Fusion FX7 Spectra multifunctional imaging system. All animal experiments were carried out in accordance with the agreement of Harbin Medical University Cancer Hospital (No: SYXK2019-001;

SCXK2019-001).

\section{Histology analysis}


After 14 days, the main tissues of mice (heart, liver, spleen, lung and kidney tumors) were collected for histological analysis to further evaluate the toxicity of EuMOF@ZIF/AP-PEG nanocomposite on tumorbearing mice and the efficacy of tumor treatment. The organs were immersed and fixed in $4 \%$ neutral formaldehyde for 14 days. After dehydration, transparency and paraffin dipping, the tissue fragments were embedded in paraffin. After solidification, the organs were cut into slices of $5 \mu \mathrm{m}$ and fixed on the slides for drying. After H\&E staining and drying in the fume hood, the tissues were examined using a microscope.

\section{Results And Discussion}

\section{Fabrication and characterization of EuMOF@ZIF}

The realization of multifunctional nanoplatforms depends on rational design of materials and successful characterization of structures. First of all, a lamellar EuMOF was prepared by solvothermal self-assembly of lanthanide europium metal ions and organic ligands of 1, 4-naphthalene dicarboxylate according to the synthesis conditions of MOF (Figure S1). A metal organic framework of zeolite imidazole was used for coating protection. $\mathrm{Zn}^{2+}$ was adsorbed onto the surface of EuMOF by electrostatic charge adsorption process. Then, through coordination between 2-MI ligand and $\mathrm{Zn}^{2+}$ ion, they reacted on the surface of EuMOF to grow ZIF, and finally synthesized the EuMOF@ZIF nanocomposite material. The EuMOF@ZIF nanocomposite was characterized in detail. Transmission electron microscopy (TEM) and Scanning electron microscopy (SEM) revealed that the particle size of EuMOF@ZIF nanocomposite was about 365 nm, and the morphology of EuMOF@ZIF nanocomposite showed the appearance of rice crust (Figure 1A). The elemental mapping showed that $\mathrm{Eu}, \mathrm{Zn}, \mathrm{C}, \mathrm{N}$ and $\mathrm{O}$ were uniformly distributed in lamellar materials (Figure 1B). Dynamic light scattering tests showed that the hydrated particle sizes of EuMOF, EuMOF-PVP and EuMOF@ZIF were about 228 nm, 253 nm and 353 nm, respectively (Figure S2, S4, 1C). Zeta potential test data showed that the potential of EuMOF, EuMOF-PVP and EuMOF@ZIF were - 13.6 $\mathrm{mV},-6.05 \mathrm{mV}$ and $+25.9 \mathrm{mV}$, respectively (Figure S3, S5, 1E). The X-ray diffraction showed that the EuMOF@ZIF nanocomposite had the same diffraction peak position as ZIF, such as degree 7, degree 11, degree 13, degree 18 have similar peak position, compared with the X-ray diffraction pattern of EuMOF given in the literature, obtained similar results (Figure 1F). In order to further confirm the group contained in the EuMOF@ZIF nanocomposite, infrared absorption test was carried out. The ligands of EuMOF contain naphthalene rings, and the characteristic skeleton vibrations of the naphthalene rings are around 1418 and $1458 \mathrm{~cm}^{-1}$. O-H, N-H stretching vibration zone in $3750-3000 \mathrm{~cm}^{-1}$; the stretching vibration zone of carbonyl group is $1900-1650 \mathrm{~cm}^{-1}$. The stretching vibration region of the double bond is $1690-1500 \mathrm{~cm}^{-}$ 1. FTIR showed that the EuMOF@ZIF nanocomposite had the same chemical bonds and groups as ZIF, and the spectral lines of EuMOF and EuMOF@ZIF were basically the same (Figure 1G). The existence of $\mathrm{Eu}, \mathrm{Zn}, \mathrm{N}$ and $\mathrm{O}$ elements was confirmed by energy spectrum test. Element $\mathrm{C}$ is a component of the carbon supporting film in transmission electron microscopy. At the same time, the table listed the weight and atomic percentage of each element. The weight percentage of Eu and $\mathrm{Zn}$ was $2.66 \%$ and $3.60 \%$ respectively, indicating that the content of $\mathrm{Zn}$ element was slightly higher than that of Eu element, which 
may be due to the dense coating of ZIF on the surface of EuMOF (Figure 1D). The UV-Vis spectrophotometric analysis showed that the absorption of EuMOF was in the band of 200-600 nm, and there were significant maximum absorption peaks at $242 \mathrm{~nm}$ and $310 \mathrm{~nm}$. Compared with EuMOF, the maximum absorption peak of EuMOF@ZIF nanocomposite had a slight red shift, but the peak pattern was basically similar (Figure 1H). The stability of EuMOF@ZIF nanocomposite was tested by sedimentation experiments in PBS solutions at different pH values, such as 7.4, 5.7 (Figure S6). EuMOF in PBS solution at pH 7.4 has obviously fast settlement after 2 h, while EuMOF@ZIF nanocomposite is quite stable in PBS solution at pH 7.4. These characterization test results indicated the successful synthesis of the nanocomposite.

\section{Microwave thermal property evaluation}

Commonly, the mechanism of microwave thermal sensitization is to convert microwave energy into energy in the form of intense collision motion through the physical action of electromagnetic response, and then convert it into heat energy by means of the dissipation action of this motion. Nanoscale MOFs and their derivatives, with its high porosity and large specific surface area, produce strong multiple scattering and reflection, and effectively improve the electromagnetic response, which have been proved to be a new generation of microwave sensitization agent [34]. In order to evaluate the microwave thermal sensitization performance of the EuMOF@ZIF nanocomposite, microwave temperature test and infrared thermal imaging real-time monitoring were carried out. The EuMOF@ZIF nanocomposite dispersed in normal saline was prepared with different concentrations $(0,2,4,8 \mathrm{mg} / \mathrm{mL})$ and irradiated by $\mathrm{MW}$ for 5 $\min \left(1.8 \mathrm{~W} \mathrm{~cm}{ }^{-2}, 450 \mathrm{MHz}\right)$. The temperature change of EuMOF, EuMOF@ZIF were monitored by infrared thermal imager (Figure S7A, 2A). A minute-by-minute snapshot of EuMOF@ZIF nanocomposite was shown below. The temperature rising curves of different concentrations of the EuMOF@ZIF nanocomposite were shown in the Figure 2B. The comparison of temperature rising of different concentrations EuMOF and EuMOF@ZIF nanocomposite was shown in the Figure 2C. The results showed that the temperature change of EuMOF@ZIF nanocomposite at $2 \mathrm{mg} / \mathrm{mL}$ was $22.7^{\circ} \mathrm{C}$, the temperature change of EuMOF@ZIF nanocomposite at $4 \mathrm{mg} / \mathrm{mL}$ was $28.1^{\circ} \mathrm{C}$, and the value change of EuMOF@ZIF nanocomposite at $8 \mathrm{mg} / \mathrm{mL}$ was $35.8^{\circ} \mathrm{C}$, respectively (Figure S7D). The temperature rising of EuMOF@ZIF nanocomposite at 2, 4, $8 \mathrm{mg} / \mathrm{mL}$ was $1.9,7.3,15^{\circ} \mathrm{C}$ higher than that of the control group, respectively. The temperature rising of EuMOF at $2,4,8 \mathrm{mg} / \mathrm{mL}$ was $4.8,4.9,9.5^{\circ} \mathrm{C}$ higher than that of the control group, respectively. The result showed the temperature rising of EuMOF@ZIF nanocomposite at $8 \mathrm{mg} / \mathrm{mL}$ significantly higher than that of EuMOF. We suspect it's because the EZ nanocomposite coated with EuMOF is more stable and difficult to agglomerate at higher concentration. Similarly, the temperature rising curve of EuMOF, the real-time IR thermal image per minute, and the temperature rise comparison histogram were also listed (Figure S7B, S7C). The microwave heating effect test showed that EuMOF@ZIF nanocomposite had excellent microwave heating performance.

\section{Luminescent property evaluation}


It has been reported that the characteristic emission wavelength of the lanthanide metal europium ion is around $618 \mathrm{~nm}$, and the emission peak is sharp. First, the emission spectra of nanomaterials at different excitation wavelengths ( $E x=280,300,320,340,360,380,390,400,420,440 \mathrm{~nm}$ ) were measured. The emission range is $500-750 \mathrm{~nm}$, the excitation end band-pass is $5 \mathrm{~nm}$, the emission end band-pass is 5 $\mathrm{nm}$, and the integration time is $0.05 \mathrm{~s}$. The emission spectrum of each sample showed that the excitation wavelength increased from $280 \mathrm{~nm}$ to $440 \mathrm{~nm}$ (Figure 2D, 2E). Whether EuMOF or EuMOF@ZIF, the intensity of the maximum emission peak at $618 \mathrm{~nm}$ gradually decreased. The relationship between the maximum emission peak intensity and excitation wavelength of EuMOF and EuMOF@ZIF was shown respectively (Figure S8 and S9). Meanwhile, fluorescence tests showed that ZIF coating did not affect the fluorescence properties of EuMOF. Then, when the excitation wavelength was $280 \mathrm{~nm}$, the solvent was $5 \%$ glucose solution, and the fluorescence intensity of the samples (EuMOF, EuMOF@ZIF) with different concentrations was tested, as shown in Figure 2G, 2H. Therefore, it is anticipated that EuMOF@ZIF nanocomposite could be a nano-fluorescent probe for tumor diagnosis.

\section{Drug loading and release}

Apatinib is a star drug approved by the US Food and Drug Administration and currently used in first-line chemotherapy. It is loaded on the EuMOF@ZIF nanocomposite to make chemotherapy and microwavethermal therapy play the role of tumor treatment synergistically. The drug loading rate was calculated by testing the characteristic UV absorption of Apatinib. The UV absorption curve was the characteristic UV absorption of apatinib in the supernatant of EuMOF@ZIF/AP (Figure S10). According to the calculation formula in the previous report of our research group, the drug loading of EZ was calculated to be $20.37 \%$, while the drug loading of EuMOF was calculated to be $9.2 \%$. It also demonstrated that the ZIF effectively improved drug-loading rate of the EuMOF@ZIF nanocomposite. In order to understand the drug release of EuMOF@ZIF/AP, drug release experiments were carried out. Ultraviolet absorption curves of apatinib drug release of different concentrations were obtained, as shown in Figure S11, and the standard curve of drug release (Figure S12) was drawn by absorption peaks of apatinib drug release of different concentrations. According to the drug release curve, the drug release rate of the EuMOF@ZIF/AP nanocomposite was calculated to be $55.68 \%$ under neutral physiological conditions (Figure 2F). In addition, the results of microwave stimulated drug release experiments showed that the drug release rate reached $84.40 \%$

(Figure 2I). The experimental results showed that EuMOF@ZIF nanocomposite could achieve loading and release of the drug, and the ZIF effectively improved drug loading rate of the EuMOF@ZIF nanocomposite.

Moreover, apatinib is known to down-regulate vascular epidermal growth factor (VEGF) expression in tumor vessels. VEGF immunofluorescence was used to test whether EZAP could down-regulate VEGF expression. Strong green fluorescence was observed in the control group, and obvious fluorescence signal was also observed in the EZP group, while the green fluorescence signal was almost not obvious in the apatinib group and EZAP group (Figure S13). The results showed that EZAP down-regulated VEGF expression, but EZP did not, further indicating the successful loading of apatinib. 


\section{In vitro microwave thermo-chemotherapy effect}

In order to better investigate the subsequent biological applications, the PEG modification of the nanocomposite was used to improve the biocompatibility and long-term circulation. First, the size distribution of EuMOF@ZIF-PEG (EZP) nanocomposite was tested as 349 nm, while the zeta-potential of EuMOF@ZIF-PEG nanocomposite is +14.2 mV, which implied the size of EuMOF@ZIF nanocomposites modified by PEG was negligible, but its potential decreased, indicating that the modification was successful. (Figure S14, S15). Next, Hepg2 and H22 were used as tumor cell models, and L929 as normal cell models. MTT test results showed that the EuMOF@ZIF-PEG nanocomposite had good biocompatibility for both tumor and normal cells (Figure 3A). Additionally, the relative cell viability of EuMOF was tested and the results showed good biosafety (Figure S16-S18). As a drug carrier, it is endowed with the chemotherapeutic effect by the EuMOF@ZIF/AP-PEG nanocomposite obtained after loading apatinib. Firstly, the relative cell viability of the EuMOF@ZIF/AP-PEG nanocomposite on HepG2 tumor cells at different concentrations were investigated to evaluate the efficacy of chemotherapy. The experimental results were shown in Figure 3B. The relative cell viability was above $80 \%$ at $0-50 \mu \mathrm{g} / \mathrm{mL}$, which could not achieve the therapeutic effect. Compared with the EuMOF@ZIF-PEG nanocomposite, the relative cell viability decreased to $60.04 \%$ at $100 \mu \mathrm{g} / \mathrm{mL}$ and $12.53 \%$ at $200 \mu \mathrm{g} / \mathrm{mL}$, indicating that the EuMOF@ZIF/AP-PEG nanocomposite at $100 \mu \mathrm{g} / \mathrm{mL}$ had certain killing effect on tumor cells (Figure 3B). Therefore, the therapeutic dose chosen in this paper was $100 \mu \mathrm{g} / \mathrm{mL}$. The results showed that apatinib, as a chemotherapy drug, had a certain inhibitory effect on HepG2 tumor cells.

Next, we performed tumor cell inhibition experiments in vitro. Compared with the control group, the relative cell viability of free Apatinib group, EuMOF@ZIF-PEG group, MW group, EuMOF@ZIF/AP-PEG group, EuMOF@ZIF-PEG + MW group and EuMOF@ZIF/AP-PEG + MW group were 84.9\%, 81.8\% and 78.5, $63.8 \%, 45.1 \%, 31.0 \%$, respectively (Figure $3 C$ ). The results showed that EuMOF@ZIF/AP-PEG + MW group had the best tumor inhibition effect. Under microwave irradiation, it showed the combination of microwave sensitization of EuMOF@ZIF/AP-PEG and chemical therapy. In addition, H22 tumor cell inhibition experiments were conducted, as shown in Figure S19 and S20. Compared with the control group, the relative cell viability of EuMOF@ZIF-PEG group, EuMOF@ZIF/AP-PEG group, MW group, EuMOF@ZIF-PEG + MW group, and EuMOF@ZIF/AP-PEG + MW group were 88.7\%, 88.5\%, 66.1\%, 36.3\%, $12.3 \%$, respectively, the experimental results showed that similar results were obtained compared with the HepG2 experiment.

To further verify the inhibitory effect of composite nanomaterials on HepG2 cells and $\mathrm{H} 22$ cells by the Calcein-AM/PI staining. The experimental results of HepG2 cells were shown in Figure 3D. First, the red fluorescence in the control group is the least, indicating the least number of dead cells. Secondly, the red fluorescence intensity of free Apatinib group and EuMOF@ZIF-PEG group was almost the same, but stronger than that of the control group. In addition, the fluorescence intensity of the MW group and the EuMOF@ZIF/AP-PEG group were similar, but the fluorescence intensity of the MW group and the EuMOF@ZIF/AP-PEG group was significantly increased compared with the previous two groups. Finally, compared with the other groups, bright and intense red fluorescence could be seen in EuMOF@ZIF/AP- 
PEG + MW group, followed by the EuMOF@ZIF-PEG + MW group, indicating the highest killing efficiency in the EuMOF@ZIF/AP-PEG + MW group. In the Calcein-AM staining of living cells, compared with the control group, the green fluorescence intensity of free Apatinib group, EuMOF@ZIF-PEG group, MW group, EuMOF@ZIF/AP-PEG group, EuMOF@ZIF-PEG + MW group, and EuMOF@ZIF/AP-PEG + MW group were in order. The fluorescence intensity of EuMOF@ZIF/AP-PEG + MW group decreased most obviously, indicating that the number of surviving cells was the least. Similar data were obtained from the staining experiment for HepG2 cells and the staining experiment for suspension H22 cells (Figure S21). In general, the results of Calcein-AM/PI live staining showed that EuMOF@ZIF/AP-PEG + MW group had the strongest tumor inhibition effect, which reflected the microwave sensitization of the EuMOF@ZIF/AP-PEG nanocomposite and the combined effect of chemical therapy under microwave irradiation.

\section{In vivo liver cancer microwave thermo-chemotherapy}

The above results showed that EuMOF@ZIF/AP-PEG nanocomposite was designed and characterized, which possessed high microwave sensitization, fluorescence imaging function and good drug loading in vitro. In order to verify the above properties of the material in vivo, $\mathrm{H} 22$ tumor-bearing mice were used as animal experimental model to evaluate the tumor inhibition effect. All animal experiments were carried out in accordance with the agreement of Harbin Medical University Cancer Hospital. Next, the experimental design was divided into 6 groups: control group (without any treatment), EuMOF@ZIF/APPEG group (containing drug material), free Apatinib group, MW group, EuMOF@ZIF-PEG + MW group (without drug material and microwave), and EuMOF@ZIF/AP-PEG + MW group (containing drug material and microwave). The changes of body weight showed that the body weight of mice in each group did not fluctuate much within 15 days (Figure 4C), demonstrating the biocompatibility and biosafety of the nanocomposite in vivo. During microwave irradiation, the tumor temperature changes in the MW group, EuMOF@ZIF-PEG + MW group and EuMOF@ZIF/AP-PEG + MW group were monitored in real time. With the increase of microwave irradiation time, the tumor temperature in each group increased, and it can be seen that the temperature rise of the experimental group was obviously higher than that of the control group. It is further proved that the EuMOF@ZIF/AP-PEG nanocomposite has good microwave sensitization performance under microwave irradiation (Figure 4A, 4B). The tumor volume of mice in the EuMOF@ZIF/AP-PEG+MW group significantly less than the control group after 14 days (Figure 4D). Moreover, the inhibitory rate of EuMOF@ZIF/AP-PEG+MW group was $98.5 \%$ compared with the control group according to the formula of inhibitory rate (Figure 4E), and the results of tumor inhibition experiment in vivo showed that the EuMOF@ZIF /AP-PEG+MW group had a good tumor inhibition effect. Images of different groups of tumor-bearing mice at 0 and 14 days, and tumor tissue sections in vitro (Figure S22 and $4 \mathrm{H}$ ) showed that the MW group, the drug-containing material without microwave and the simple material plus microwave group are higher than the control group, but the therapeutic effect is lower than the experimental group, indicating that microwave-thermal therapy combined with chemotherapy could effectively inhibit tumor growth and enhance the effect of microwave treatment. In addition, as shown in Figure $\mathbf{4 F}$ and $\mathbf{4 G}$, the survival rate of both EZAP + MW group and EZP + MW group was $100 \%$, the survival rate of the MW group was $66.6 \%$, the survival rate of the EZAP group and the AP 
group was $33.3 \%$, and the survival rate of the control group was $0 \%$. In a word, in vivo tumor inhibition experiment results showed that thermal therapy combined with chemotherapy under microwave irradiation could achieve a good effect on tumor treatment.

In order to further observe and analyze the biological safety of the nanocomposite in mice, Hematoxylin eosin staining was performed (Figure 5). The results showed that there was no obvious organ damage in the tissue sections of heart, liver, spleen, lung and kidney tumors in each group. However, in tumor tissue, the EuMOF@ZIF/AP-PEG+MW group had significant large area of cell damage, followed by the EuMOF@ZIF-PEG+MWgroup, EuMOF@ZIF/AP-PEG group and MW group had a small amount of cell damage, and the control group had no cell damage.

\section{In vivo fluorescence imaging}

Encouraged by the fluorescence properties of the EuMOF@ZIF nanocomposite mentioned above, we performed fluorescence imaging monitoring of mouse tumors in vivo. The emission spectra of the EuMOF@ZIF-PEG nanocomposite at different excitation wavelengths were studied. The emission map of the EuMOF@ZIF-PEG nanocomposite showed that the intensity of the maximum emission peak at $618 \mathrm{~nm}$ gradually decreased as the excitation wavelength gradually increased. In addition, the relationship between the maximum emission peak value and the excitation wavelength was plotted (Figure S23, S24), the fluorescence test showed that PEG modification did not affect the fluorescence properties of the EuMOF@ZIF nanocomposite. Furthermore, fluorescence signal images of different material concentrations were obtained in in vivo imaging experiments (Figure 6A). As well as the fluorescence signal images collected at different time points after the injection of EuMOF@ZIF/AP-PEG nanocomposite into the tail vein of mice (Figure 6B). The signal of the EuMOF@ZIF/AP-PEG nanocomposite in mouse tumor was enhanced with the extension of time after the injection of caudal vein in a certain period of time. At $6 \mathrm{~h}$, there were obvious fluorescence signals in the tumor sites of mice. Then, with the passage of time, the fluorescence signals of the EuMOF@ZIF/AP-PEG nanocomposite in the tumor sites of mice gradually decreased. To understand the effect of composite nanomaterials on the organs of mice, we dissected the main organs and tumors of mice at different time points to observe the change of fluorescence signal intensity. The fluorescence signal at the tumor site was the strongest after $6 \mathrm{~h}$ material injection (Figure 6C), which was consistent with the experimental results in vivo. In addition, there were also obvious fluorescence signals in the liver and lung of mice, followed by the kidney and heart, and the spleen. In general, the EuMOF@ZIF/AP-PEG nanocomposite as fluorescent probes had demonstrated their application prospects in vivo imaging and demonstrated their diagnostic potential.

\section{Conclusions}

In summary, a novel theranostic nanoplatform (EuMOF@ZIF/AP-PEG nanocomposite) was designed and characterized, which had high microwave sensitization, fluorescence imaging function and good drug loading. MTT assay showed that the concentration of EZAP nanocomposite was $100 \mu \mathrm{g} / \mathrm{mL}$ and had low 
biotoxicity. In vitro tumor cell inhibition experiments showed that thermal therapy combined with chemotherapy under microwave irradiation had the strongest inhibitory effect on tumor cell growth. Compared with $100 \%$ in the control group, the relative activity of cells was reduced to $31 \%$. Through the treatment experiment of $\mathrm{H} 22$ tumor-bearing mice, it was further verified that the EZAP nanocomposite had good microwave sensitization. The EZAP nanocomposite assisted MW irradiation, thermal therapy combined with chemotherapy could achieve a good effect on tumor treatment, showing excellent antitumor effect, tumor inhibition rate of $98.5 \%$, indicating that the EZAP nanocomposite had a good potential for the combined treatment of microwave-thermal therapy and chemotherapy. The fluorescence imaging in vivo experiment showed that the EZAP nanocomposite could be used as a potential fluorescent probe. The EZAP nanocomposite showed was strongest fluorescence signal after $6 \mathrm{~h}$ of caudal vein injection, which could monitor the treatment of tumor and realize the integrated function of diagnosis and treatment. Our design strategy advances the lanthanide MOF in vivo imaging research. It also paves a novel way for the biological application of lanthanide MOF.

Taken together, the EZAP nanocomposite demonstrated the potential of microwave thermochemotherapy therapy and in vivo imaging, and provided further inspiration for future imaging monitoring therapies.

\section{Declarations}

\section{Authors' contributions}

LZ: methodology, conceptualization, investigation, data curation, methodology, formal analysis, validation, writing original draft. WZ: data curation, methodology, resources. QW: resources, software, resources, supervision. CF: investigation, software, resources, supervision. KL: methodology, funding acquisition. TM: methodology, super-vision, resources. XC: conceptualization, funding acquisition, supervision, project administration. LT: investigation, software, resources, supervision. XM: conceptualization, funding acquisition, writing review \& editing, supervision, project administration. All authors read and approved the final manuscript.

\section{Funding}

We acknowledge financial support from the National Natural Science Foundation of China (U20A20335, 91859201, 41903056), Beijing Natural Science Foundation (7212208), Shenzhen Science and Technology Foundation JCYJ20180305163851409, the Sichuan Science and Technology Program (2020YFSY0018).

\section{Availability of data and materials}

The datasets and materials used in the study are available from the corresponding author.

\section{Ethics approval and consent to participate}


All animal experiments were carried out in accordance with the agreement of Harbin Medical University Cancer Hospital (No: SYXK2019-001; SCXK2019-001). All the animal experimental operations were in compliance with the National Guidelines for Animal Protection.

\section{Consent for publication}

All authors have approved the manuscript be submitted.

\section{Competing Interests}

The authors declare no competing financial interest.

\section{Author details}

${ }^{1}$ Laboratory of Controllable Preparation and Application of Nanomaterials, CAS Key Laboratory of Cryogenics, Technical Institute of Physics and Chemistry, Chinese Academy of Sciences, No. 29 East Road Zhongguancun, Beijing 100190, P. R. China. ${ }^{2}$ Department of Nuclear Medicine, Harbin Medical University Cancer Hospital, Nangang District, Harbin, Heilongjiang Province 150086, P.R.

China. ${ }^{3}$ Department of Interventional Radiology, Shenzhen People's Hospital (The Second Clinical Medical College, Jinan University; The First Affiliated Hospital, Southern University of Science and Technology), Shenzhen 518020, Guangdong, China. ${ }^{4}$ University of Chinese Academy of Sciences, Beijing 100049, P.R. China.

\section{References}

1. Chu K F, Dupuy D E. Thermal ablation of tumours: biological mechanisms and advances in therapy. Nat. Rev. Cancer. 2014; 14: 199-208.

2. Ahmed M, Brace C L, Lee F T,Goldberg S N. Principles of and Advances in Percutaneous Ablation. Radiology. 2011; 258: 351-369.

3. Couri T,Pillai A. Goals and targets for personalized therapy for HCC. Hepatol. Int. 2019; 13: 125-137.

4. Izzo F, Granata V, Grassi R, Fusco R, Palaia R, Delrio P, et al. Radiofrequency Ablation and Microwave Ablation in Liver Tumors: An Update. Oncologist. 2019; 24: e990-e1005.

5. Ni Y, Ye X, Yang X, Huang G H, Li W H, Wang J, et al. Microwave ablation as local consolidative therapy for patients with extracranial oligometastatic EGFR-mutant non-small cell lung cancer without progression after first-line EGFR-TKIs treatment. J. Cancer Res. Clin. Oncol. 2020; 146: 197203.

6. Paulides M M, Trefna HD, Curto S,Rodrigues D B. Recent technological advancements in radiofrequency- and microwave-mediated hyperthermia for enhancing drug delivery. Adv. Drug Deliv. Rev. 2020; 163: 3-18.

7. Lachenmayer A, Tinguely P, Maurer M H, Frehner L, Knpfli M, Peterhans M, et al. Stereotactic imageguided microwave ablation of hepatocellular carcinoma using a computer-assisted navigation 
system. Liver Int. 2019; 39: 1975-1985.

8. Chen X W, Fu C H, Wang Y Q, Wu Q R, Meng X W,Xu K. Mitochondria-targeting nanoparticles for enhanced microwave ablation of cancer. Nanoscale. 2018; 10: 15677-15685.

9. Chen Z Z, Niu M, Chen G, Wu Q, Tan L F, Fu C H, et al. Oxygen Production of Modified Core-Shell CuO@ZrO2 Nanocomposites by Microwave Radiation to Alleviate Cancer Hypoxia for Enhanced Chemo-Microwave Thermal Therapy. ACS Nano. 2018; 12: 12721-12732.

10. Li W S, Wang X J, Zhang S, Hu J B, Du Y L, Kang X Q, et al. Mild microwave activated, chemo-thermal combinational tumor therapy based on a targeted, thermal-sensitive and magnetic micelle. Biomaterials. 2017; 131: 36-46.

11. Chen Z Z, Guo W N, Wu Q, Tan L F, Ma T C, Fu C H, et al. Tumor reoxygenation for enhanced combination of radiation therapy and microwave thermal therapy using oxygen generation in situ by CuO nanosuperparticles under microwave irradiation. Theranostics. 2020; 10: 4659-4675.

12. Shi H T, Niu M, Tan L F, Liu T L, Shao H B, Fu C H, et al. A smart all-in-one theranostic platform for CT imaging guided tumor microwave thermotherapy based on IL@ZrO 2 nanoparticles. Chem. Sci. 2015; 6: 5016-5026.

13. Wu B, Xia N, Long D, Tan L F, Rao W, Tu J, et al. Dual-Functional Supernanoparticles with Microwave Dynamic Therapy and Microwave Thermal Therapy. Nano Lett. 2019; 19: 5277-5286.

14. Xu J S, Chen X Q, Tan L F, Fu C H, Ahmed M, Tian J, et al. Microwave Responsive Nanoplatform via PSelectin Mediated Drug Delivery for Treatment of Hepatocellular Carcinoma with Distant Metastasis. Nano Lett. 2019; 19: 2914-2927.

15. Du Q J, Fu C H, Tie J, Liu T L, Li L L, Ren X L, et al. Gelatin microcapsules for enhanced microwave tumor hyperthermia. Nanoscale. 2015; 7: 3147-3154.

16. Fu C H, He F, Tan L F, Ren X L, Zhang W, Liu T L, et al. MoS 2 nanosheets encapsulated in sodium alginate microcapsules as microwave embolization agents for large orthotopic transplantation tumor therapy. Nanoscale. 2017; 9: 14846-14853.

17. Tang T Y, Xu X M, Wang Z W, Tian J J, Yang Y, Ou C Z, et al. $\mathrm{Cu}_{2} Z n$ SnS $_{4}$ nanocrystals for microwave thermal and microwave dynamic combination tumor therapy. Chem. Commun. 2019; 55: 1314813151.

18. Wu S S, Zhang D Y, Yu J, Dou J P, Li X, Mu M J, et al. Chemotherapeutic Nanoparticle-Based Liposomes Enhance the Efficiency of Mild Microwave Ablation in Hepatocellular Carcinoma Therapy. Front. Pharmacol. 2020; 11: 9.

19. Long D, Niu M, Tan L F, Fu C H, Ren X L, Xu K, et al. Ball-in-ball ZrO ${ }_{2}$ nanostructure for simultaneous CT imaging and highly efficient synergic microwave ablation and tri-stimuli-responsive chemotherapy of tumors. Nanoscale. 2017; 9: 8834-8847.

20. Sun M R, Kiourti A, Wang H, Zhao S T, Zhao G, Lu X B, et al. Enhanced Microwave Hyperthermia of Cancer Cells with Fullerene. Mol. Pharm. 2016; 13: 2184-2192. 
21. Chall A, Stagg J, Mixson A, Gato E, Quirino R,Sittaramane V. Ablation of cells in mice using antibodyfunctionalized multiwalled carbon nanotubes (Ab-MWCNTs) in combination with microwaves. Nanotechnology. 2021; 32: 195102.

22. Panwar N, Soehartono A M, Chan K K, Zeng S W, Xu G X, Qu J L, et al. Nanocarbons for Biology and Medicine: Sensing, Imaging, and Drug Delivery. Chem. Rev. 2019; 119: 9559-9656.

23. Su L H, Wu Q, Tan L F, Huang Z B, Fu C H, Ren X L, et al. High Biocompatible ZIF-8 Coated by ZrO2 for Chemo-microwave Thermal Tumor Synergistic Therapy. ACS Appl. Mater. Interfaces. 2019; 11: 10520-10531.

24. Fu C H, Zhou H Q, Tan L F, Huang Z B, Wu Q, Ren X L, et al. Microwave-Activated Mn-Doped Zirconium Metal Organic Framework Nanocubes for Highly Effective Combination of Microwave Dynamic and Thermal Therapies Against Cancer. ACS Nano. 2018; 12: 2201-2210.

25. Li T, Wu Q, Wang W, Chen Z Z, Tan L F, Yu J, et al. MOF-derived nano-popcorns synthesized by sonochemistry as efficient sensitizers for tumor microwave thermal therapy. Biomaterials. 2020; 234 : 12.

26. Chen Y, Li J X, Shu N, Zheng S J, Ma M, Zhao Z B, et al. A polymeric nanoformulation improves the bioavailability and efficacy of sorafenib for hepatocellular carcinoma therapy. Biomater. Sci. 2021; 9 : 2508-2518.

27. Li L L, Xing H, Zhang J J,Lu Y. Functional DNA Molecules Enable Selective and Stimuli-Responsive Nanoparticles for Biomedical Applications. Acc. Chem. Res. 2019; 52: 2415-2426.

28. Qi J, Li W S, Lu K J, Jin F Y, Liu D, Xu X L, et al. pH and Thermal Dual-Sensitive Nanoparticle-Mediated Synergistic Antitumor Effect of Immunotherapy and Microwave Thermotherapy. Nano Lett. 2019; 19: 4949-4959.

29. Yun B F, Zhu H Q, Yuan J X, Sun Q,Li Z. Synthesis, modification and bioapplications of nanoscale copper chalcogenides. J. Mat. Chem. B. 2020; 8: 4778-4812.

30. Zhang Y R, Lin R, Li H J, He W L, Du J Z,Wang J. Strategies to improve tumor penetration of nanomedicines through nanoparticle design. Wiley Interdiscip. Rev. Nanomed. Nanobiotechnol. 2019; 11: e1519.

31. Liu Y, Zhao Y L,Chen X Y. Bioengineering of Metal-organic Frameworks for Nanomedicine. Theranostics. 2019; 9: 3122-3133.

32. Liu J T, Huang J, Zhang L,Lei J P. Multifunctional metal-organic framework heterostructures for enhanced cancer therapy. Chem. Soc. Rev. 2021; 50: 1188-1218.

33. Seaberg J, Montazerian H, Hossen M N, Bhattacharya R, Khademhosseini A,Mukherjee P. Hybrid Nanosystems for Biomedical Applications. ACS Nano. 2021; 15: 2099-2142.

34. Shu J C, Cao W Q,Cao M S. Diverse Metal-Organic Framework Architectures for Electromagnetic Absorbers and Shielding. Adv. Funct. Mater. 2021; 31: 2100470.

35. Cui Y J, Chen B L,Qian G D. Lanthanide metal-organic frameworks for luminescent sensing and lightemitting applications. Coord. Chem. Rev. 2014; 273: 76-86. 
36. Yan B. Lanthanide-Functionalized Metal-Organic Framework Hybrid Systems To Create Multiple Luminescent Centers for Chemical Sensing. Acc. Chem. Res. 2017; 50: 2789-2798.

37. Chen Y, Ye D L, Wu M Y, Chen H R, Zhang L L, Shi J L, et al. Break-up of Two-Dimensional $\mathrm{MnO}_{2}$ Nanosheets Promotes Ultrasensitive pH-Triggered Theranostics of Cancer. Adv. Mater. 2014; 26 : 7019-7026.

38. Duan X D, Wang C, Shaw J C, Cheng R, Chen Y, Li H L, et al. Lateral epitaxial growth of twodimensional layered semiconductor heterojunctions. Nat. Nanotechnol. 2014; 9: 1024-1030.

39. Hu R Z, Dai C, Wang C M, Lin J, Hu H, Li Z F, et al. Engineering 2D Arsenic-Phosphorus Theranostic Nanosheets. Adv. Funct. Mater. 2021; 31: 2101660.

40. Gorai T, Schmitt W,Gunnlaugsson T. Highlights of the development and application of luminescent lanthanide based coordination polymers, MOFs and functional nanomaterials. Dalton Trans. 2021; 50: 770-784.

41. Rocha J, Carlos L D, Paz F A A,Ananias D. Luminescent multifunctional lanthanides-based metalorganic frameworks. Chem. Soc. Rev. 2011; 40: 926-940.

42. Younis S A, Bhardwaj N, Bhardwaj S K, Kim K H,Deep A. Rare earth metal-organic frameworks (REMOFs): Synthesis, properties, and biomedical applications. Coord Chem Rev. 2021; 429: 213620.

43. Li B, Wen H M, Cui Y J, Qian G D,Chen B L. Multifunctional lanthanide coordination polymers. Prog. Polym. Sci. 2015; 48: 40-84.

44. Fang C, Cen D, Wang Y F, Wu Y J, Cai X J, Li X, et al. ZnS@ZIF-8 core-shell nanoparticles incorporated with ICG and TPZ to enable H 2 S-amplified synergistic therapy. Theranostics. 2020; 10: 7671-7682.

45. Shao F Y, Wu Y F, Tian Z Y, Liu S Q. Biomimetic nanoreactor for targeted cancer starvation therapy and cascade amplificated chemotherapy. Biomaterials. 2021; 274: 120869.

46. Xu M R, Hu Y, Ding W P, Li F F, Lin J, Wu M, et al. Rationally designed rapamycin-encapsulated ZIF-8 nanosystem for overcoming chemotherapy resistance. Biomaterials. 2020; 258: 120308.

47. Min H, Wang J, Qi Y Q, Zhang Y L, Han X X, Xu Y, et al. Biomimetic Metal-Organic Framework Nanoparticles for Cooperative Combination of Antiangiogenesis and Photodynamic Therapy for Enhanced Efficacy. Adv. Mater. 2019; 31: 1808200.

\section{Scheme}

Schema 1 is available in supplementary section.

\section{Figures}



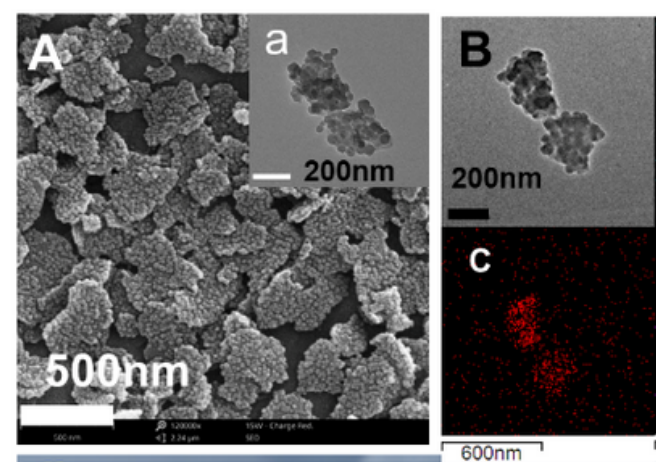

C

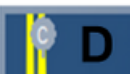

כ
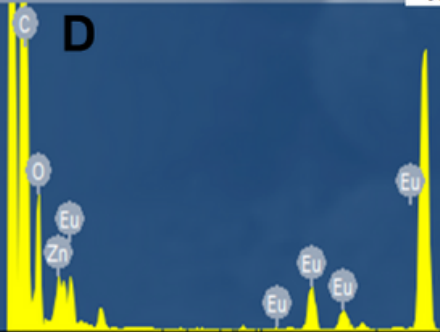

$600 \mathrm{~nm}$

Eu

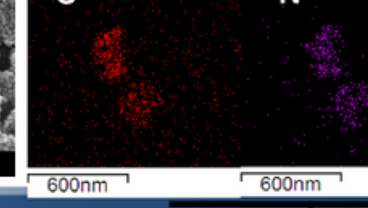

N
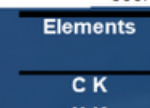

C K
N K

N K
O K
Zn K

Zn K

Eu L

Total

ois his content
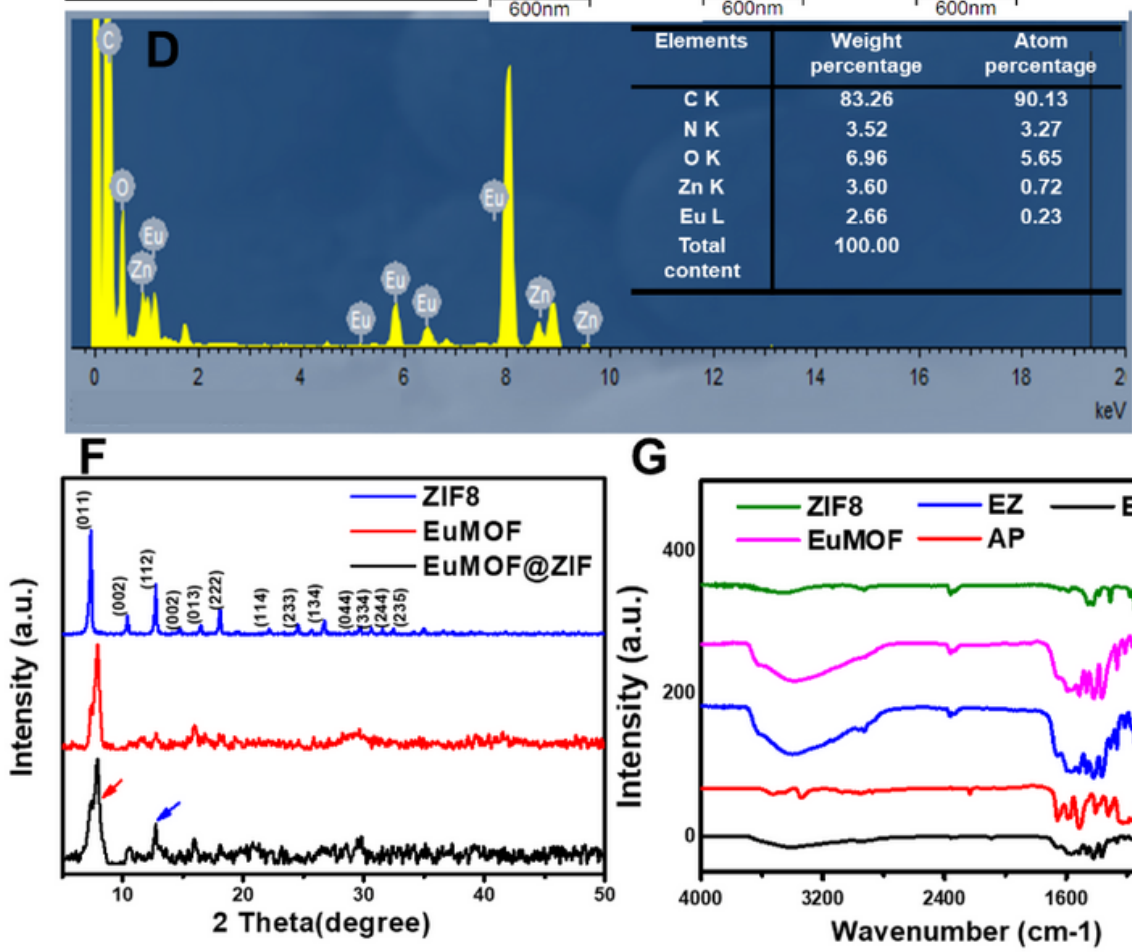

G

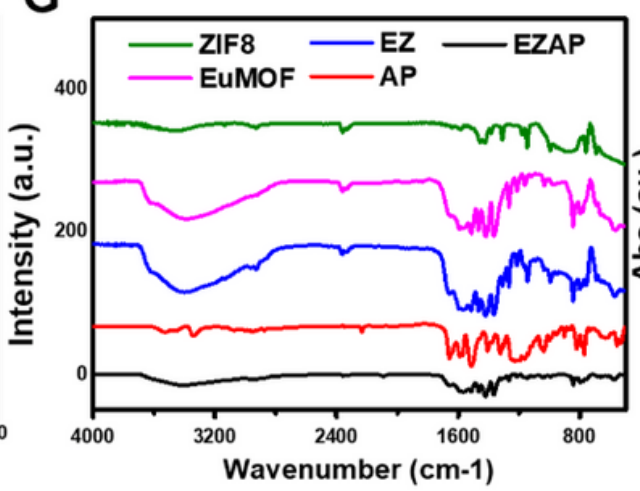

$\mathrm{H}$

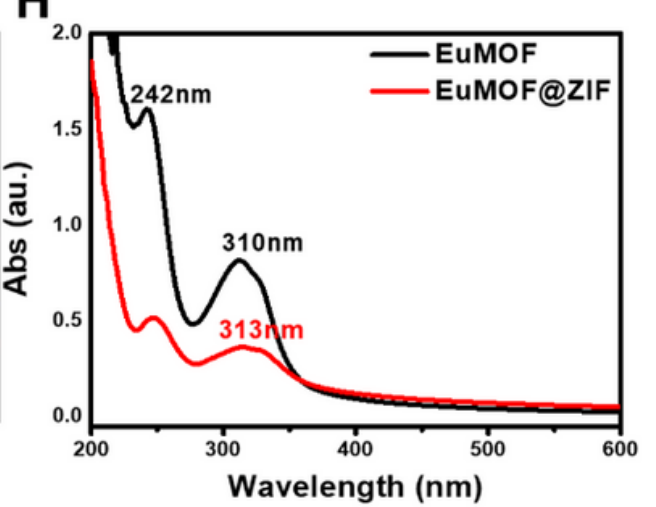

Figure 1

Characterization of EuMOF@ZIF nanocomposite. (A) SEM image of EuMOF@ZIF, TEM image of EuMOF@ZIF in the inset. (B) Elemental mapping of EuMOF@ZIF. (C) Zeta-potential of EuMOF@ZIF, particle size distribution of dynamic light scattering of EuMOF@ZIF in the inset. (D) EDS of EuMOF@ZIF, element content of EuMOF@ZIF in the inset. (E) Particle size histogram of EuMOF, EuMOF-PVP, EuMOF@ZIF, zeta-potentials of EuMOF, EuMOF-PVP, EuMOF@ZIF in the inset. (F) XRD, (G) FTIR of ZIF8, EuMOF, EuMOF@ZIF. (H) UV-vis absorption of EuMOF, EuMOF@ZIF. 

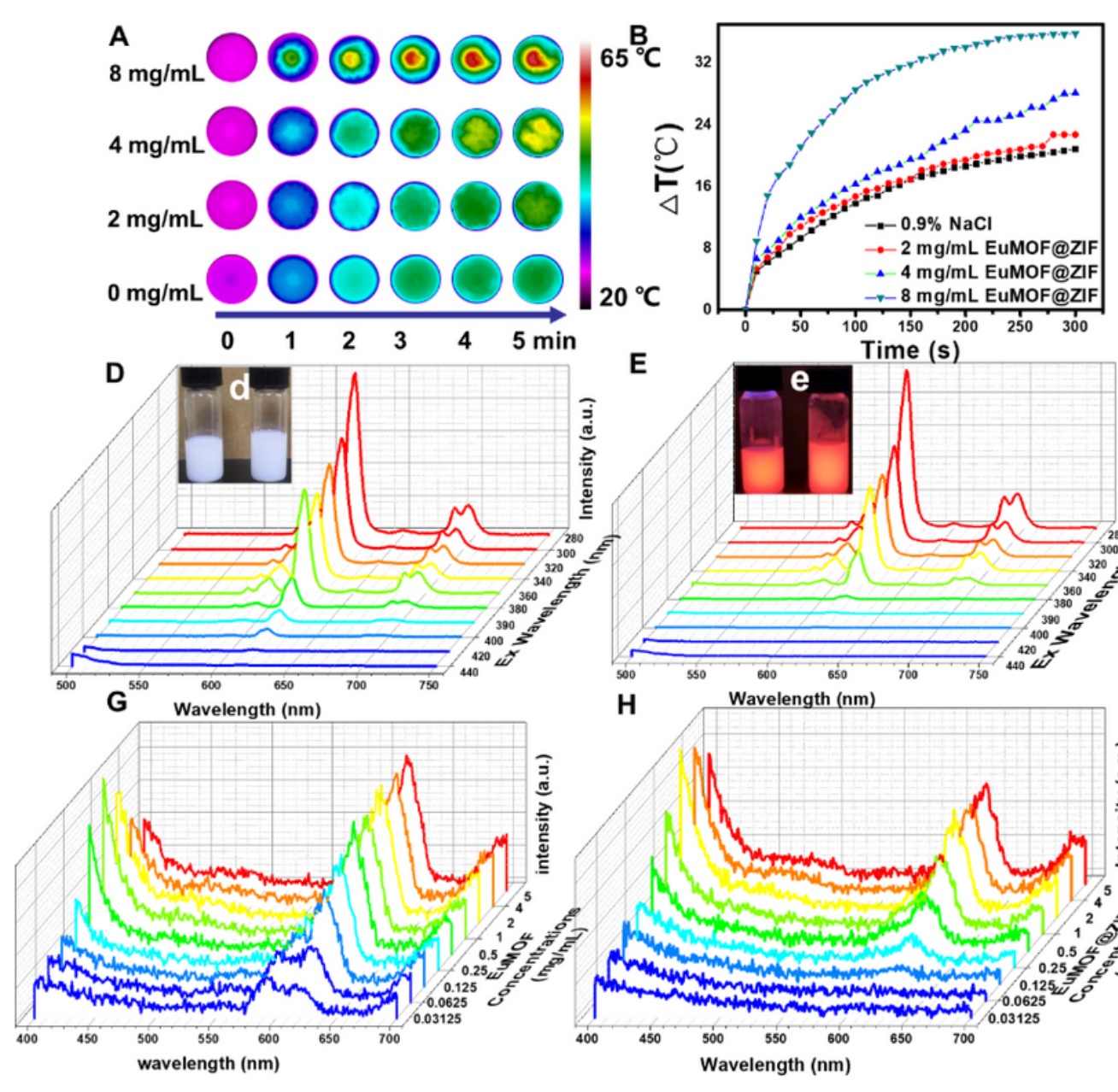

C
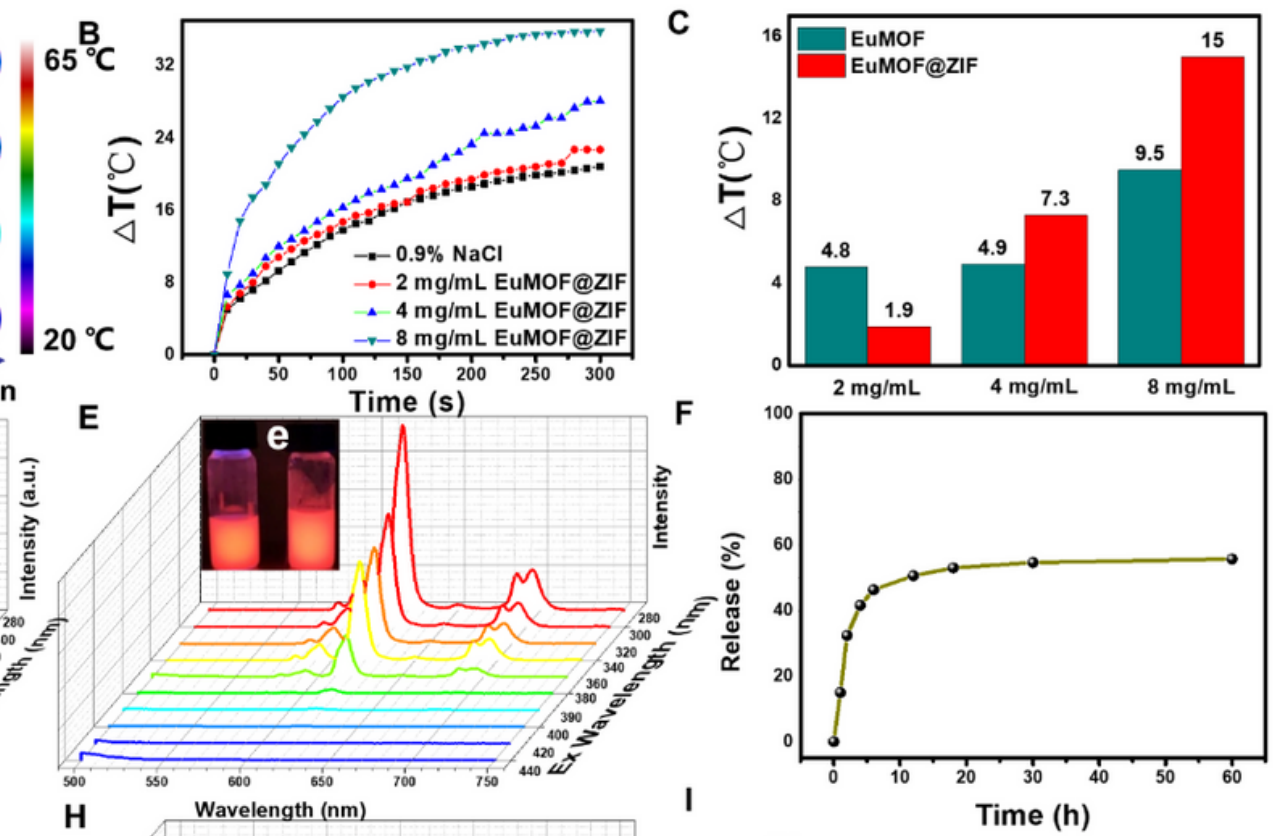

H

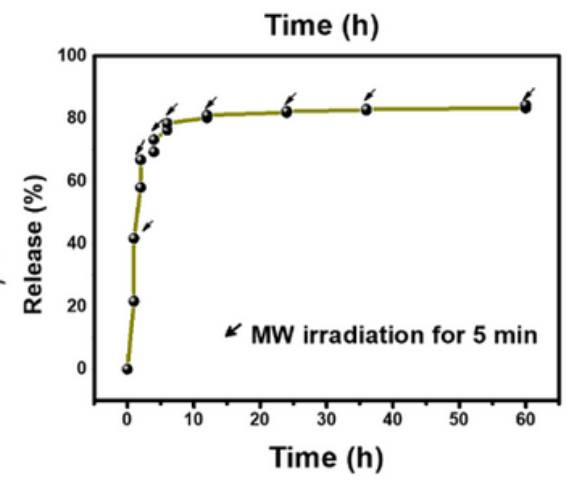

Figure 2

Microwave thermal property, luminescent property and drug loading and release of EuMOF@ZIF nanocomposite. (A) Infrared thermal images, (B) temperature variation curves, of EuMOF@ZIF (0, 2, 4, 8 $\mathrm{mg} / \mathrm{mL}$ ) under microwave irradiation for $5 \mathrm{~min}$. (C) Histogram of temperature rise between EuMOF@ZIF $(2,4,8 \mathrm{mg} / \mathrm{mL})$ and blank concentration group, as well as EuMOF. Emission spectra of (D) EuMOF, (E) EuMOF@ZIF at different excitation wavelengths, pictures in the inset from left to right of EuMOF区 EuMOF@ZIF (d) in daylight $\mathbb{( e )}$ under ultraviolet light. (F) Drug release rate of EZ loaded apatinib under neutral physiological condition. (I) Drug release rate of EZ loaded apatinib under microwave stimulation (Microwave irradiation for 5 min, 1.8 W). Emission spectra of (G) EuMOF, (H) EuMOF@ZIF at different concentrations. 

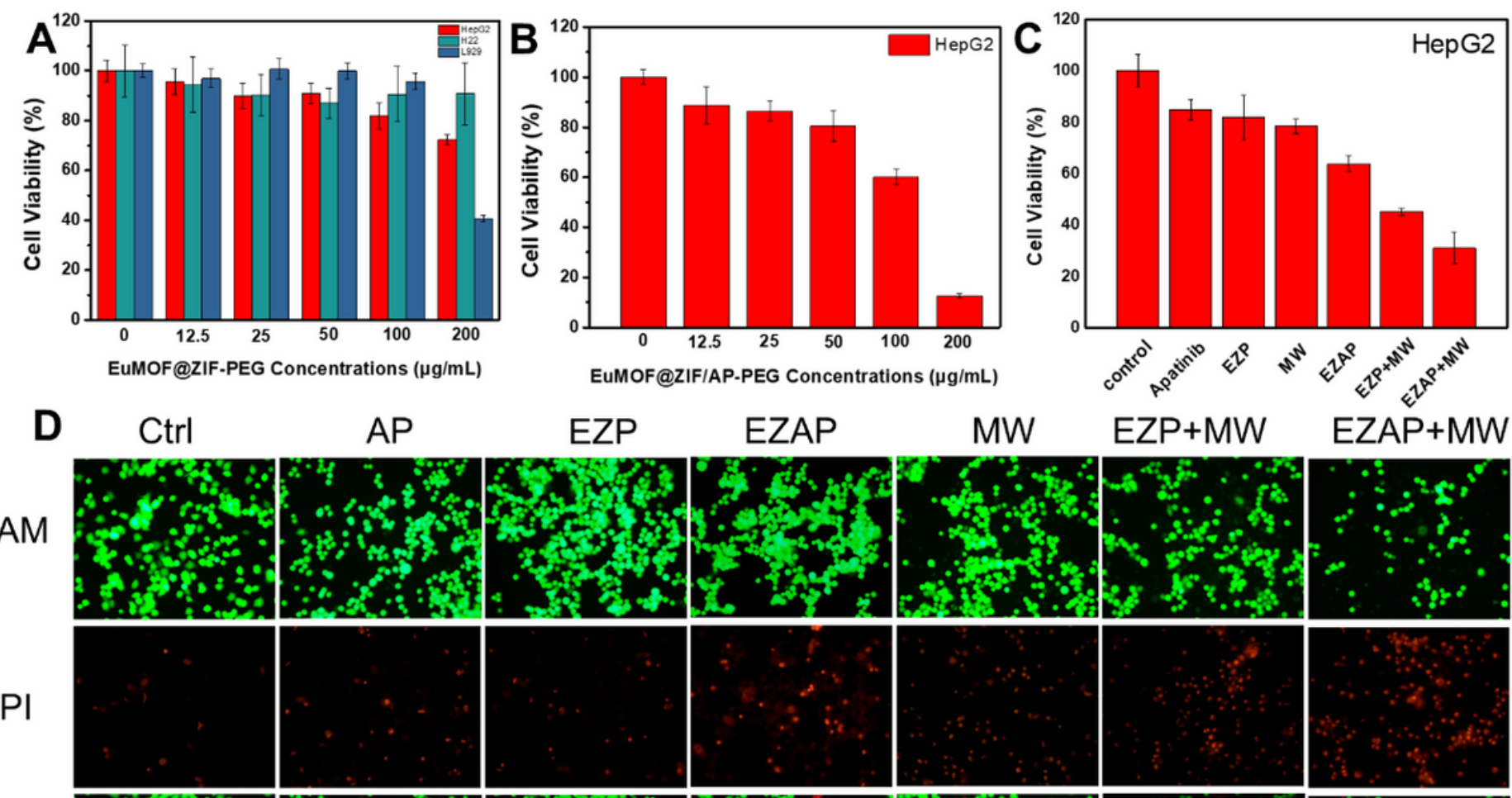

EZP

EZAP

MW

$\mathrm{EZP}+\mathrm{MW} \quad \mathrm{EZAP}+\mathrm{MW}$
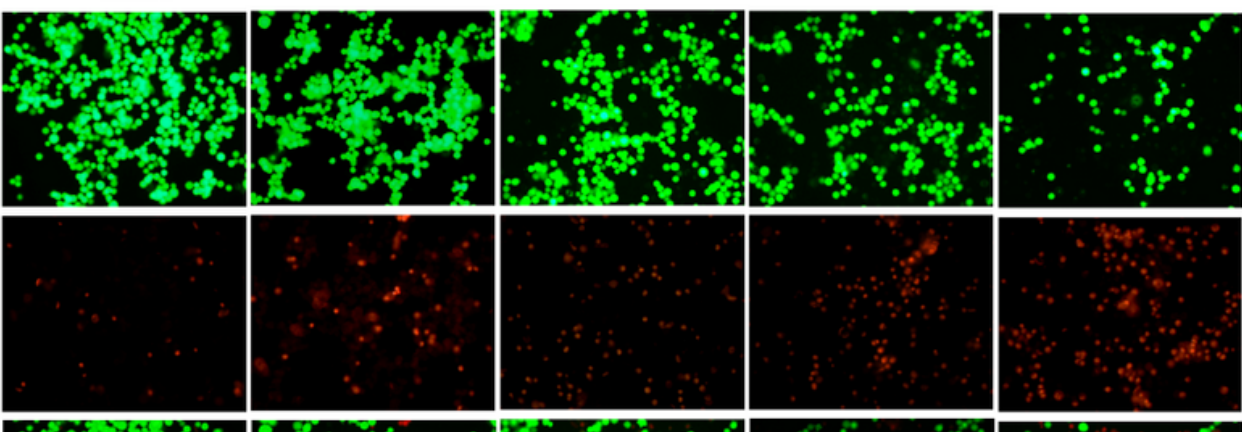

Merge
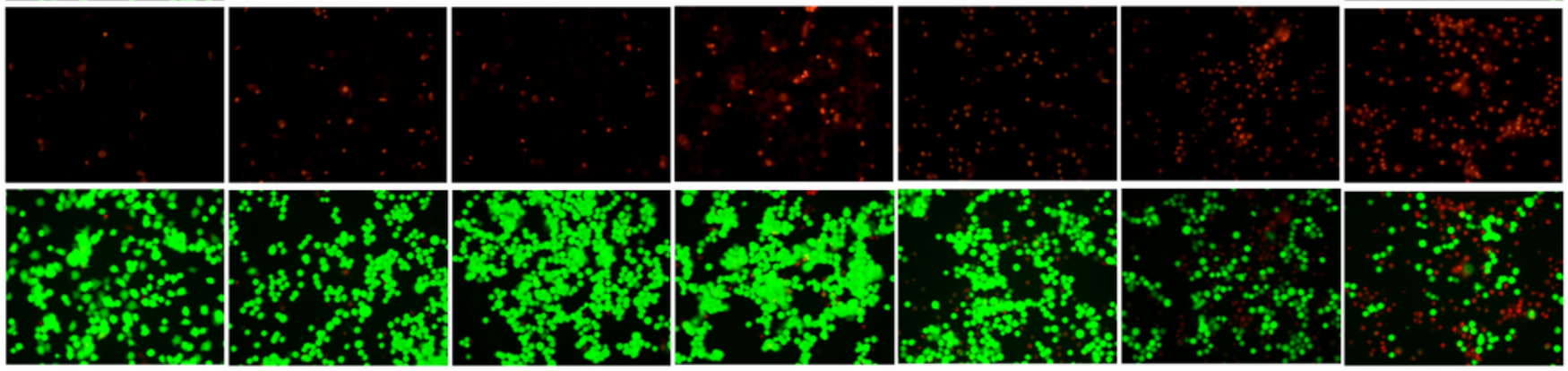

Figure 3

Cytotoxicity and in vitro microwave thermo-chemotherapy effect. (A) relative cell viability of EuMOF@ZIF after PEG modification to cancer cells and normal cells, respectively. (B) Inhibition of different concentrations of EuMOF@ZIF after drug loading on cancer cells. (C) Relative cell viability, (D) Livingdead staining of cancer cells under different treatments (control, free apatinib, EZP, MW, EZAP, EZP+MW, EZAP+MW). 


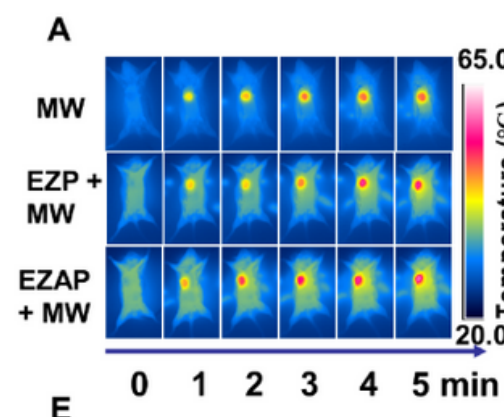

B
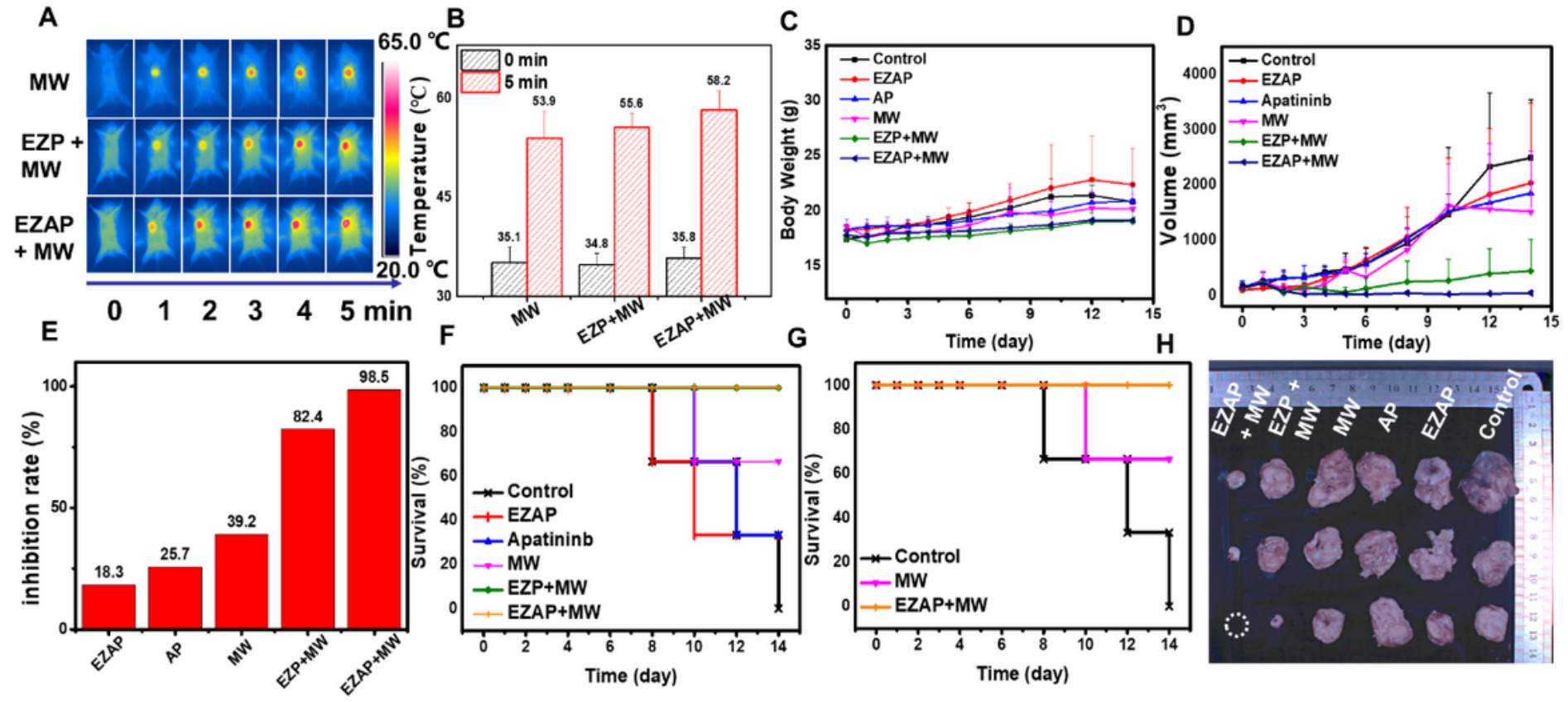

\section{Figure 4}

Microwave thermo-chemotherapy, and histology analysis. (A) Infrared thermal images of tumor-bearing mice under different treatment methods (MW, EZP + MW, EZAP + MW) under microwave irradiation for 5 min. (B) Temperature of tumor-bearing mice with different treatment methods (MW, EZP + MW, EZAP + $\mathrm{MW}$ ) at the beginning and end of microwave irradiation. (C) The body weight changes, (D) tumor volume changes of different groups of tumor-bearing mice within 15 days. (E) Inhibition rate in each group after 15 days of treatment compared to the control group. (F) Survival rates of different groups of tumorbearing mice within 15 days. (G) Survival rates of three groups (control, MW, EZAP+MW) of tumor-bearing mice within 15 days. $(\mathrm{H})$ In vitro tumor images of different groups of tumor-bearing mice on the last day are control group, EZAP group, apatinib group, MW group, EZP + MW group, and EZAP + MW group from top to bottom, the white dotted circles indicate that the mouse tumor was cured without recurrence. 


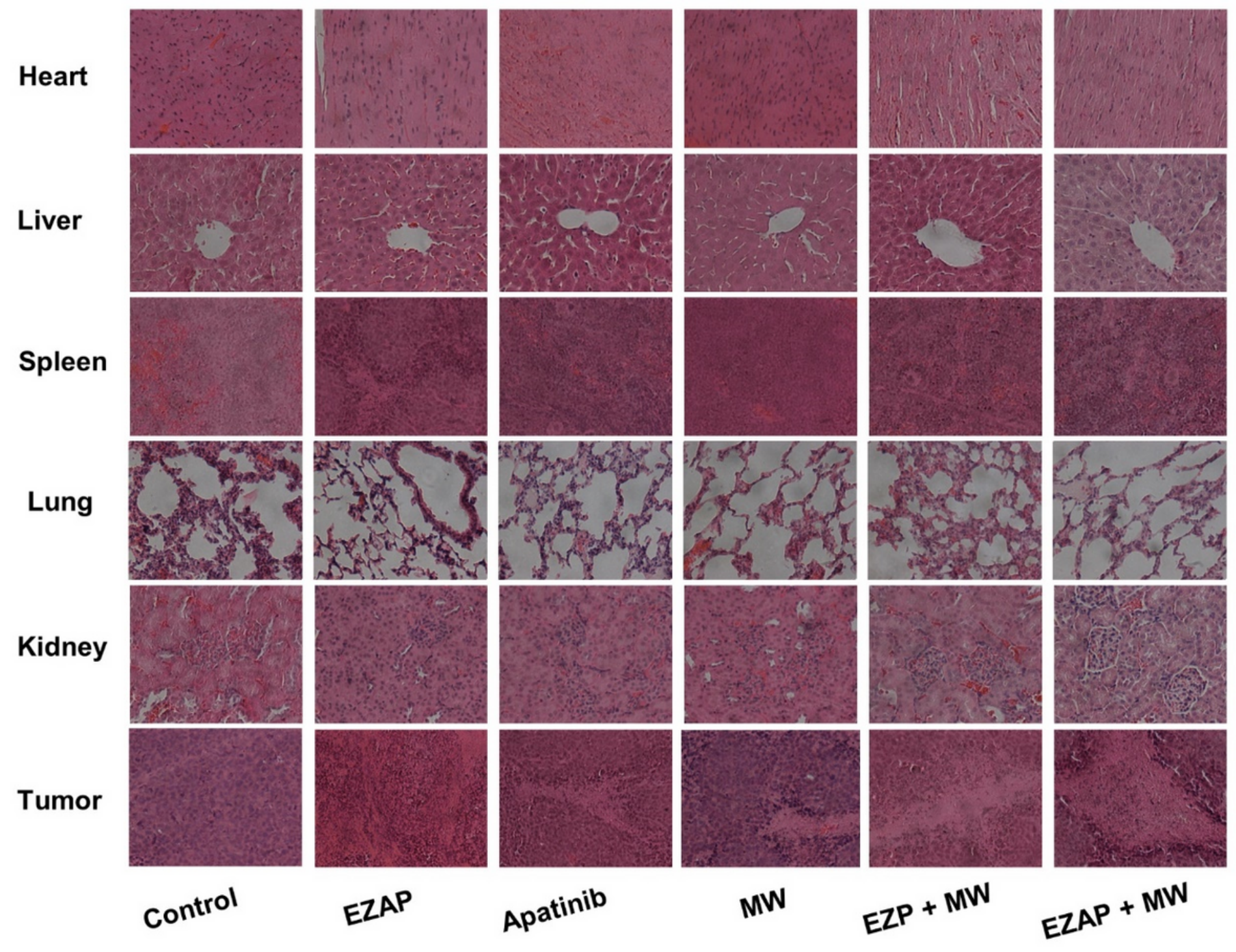

Figure 5

HE staining section of the main organs and tumors of tumor-bearing mice. 

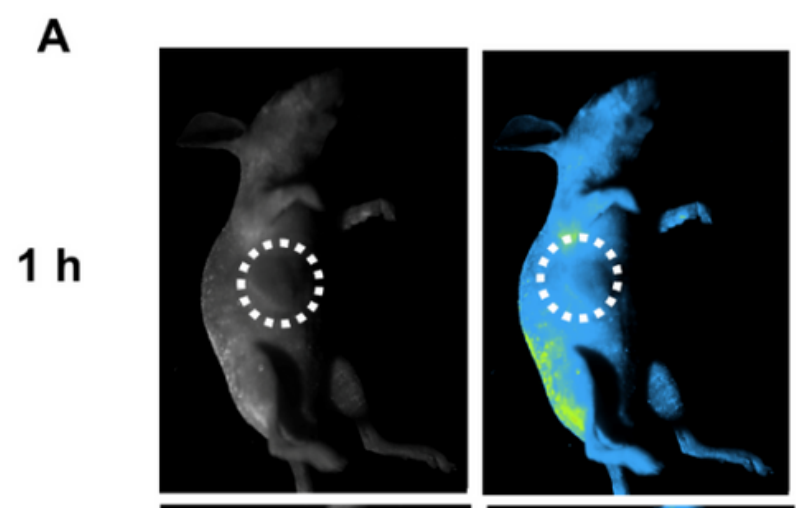

$6 \mathrm{~h}$
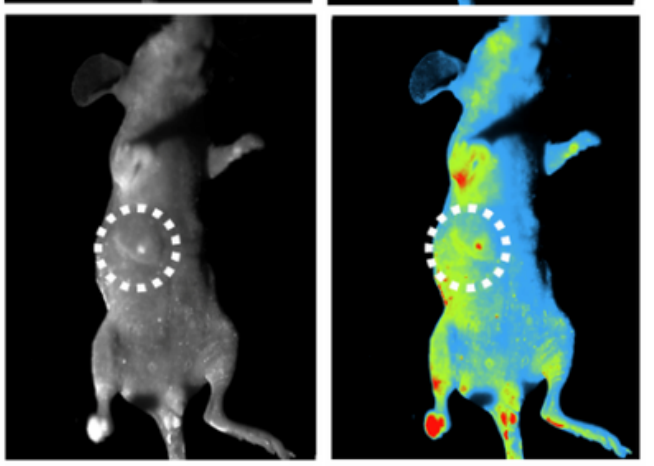

$12 \mathrm{~h}$
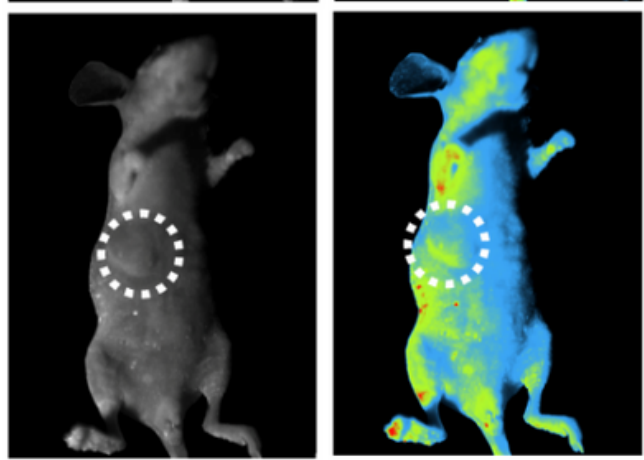

$24 \mathrm{~h}$

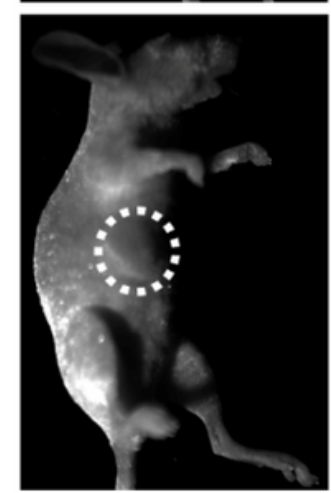

B

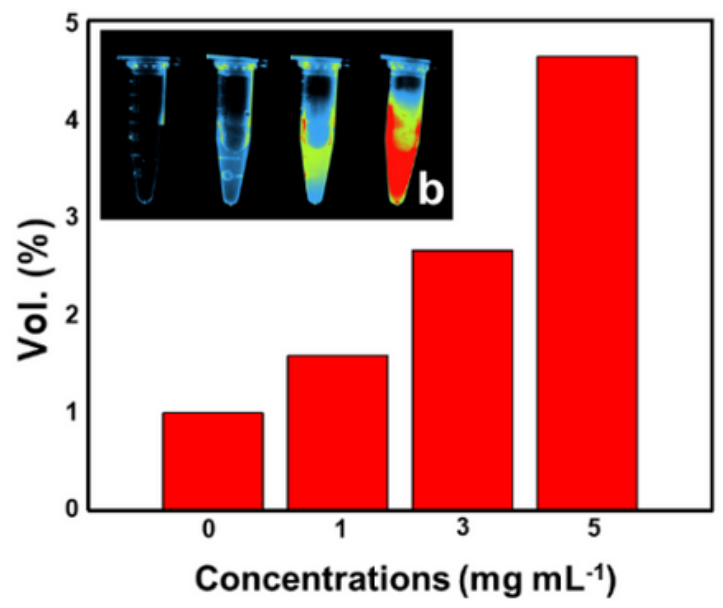

C
$2 h$
$6 h$
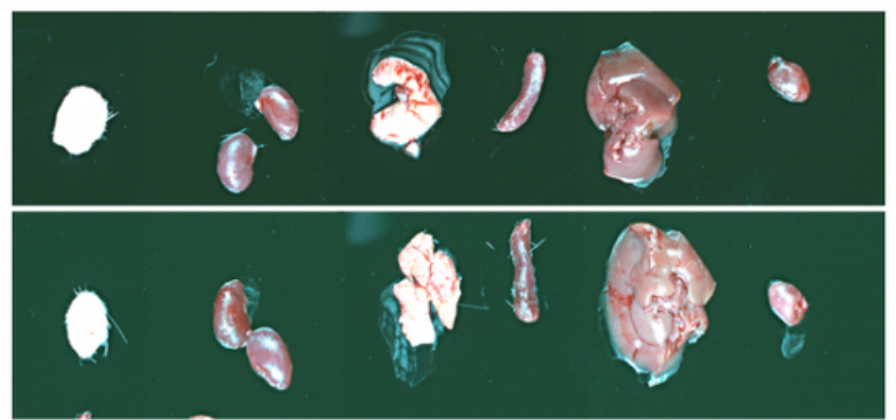

$24 \mathrm{~h}$

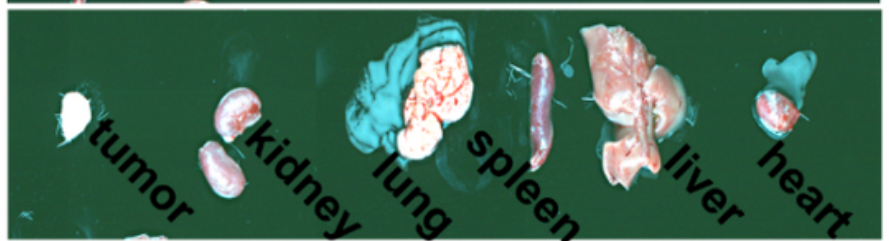

$2 \mathbf{h}$

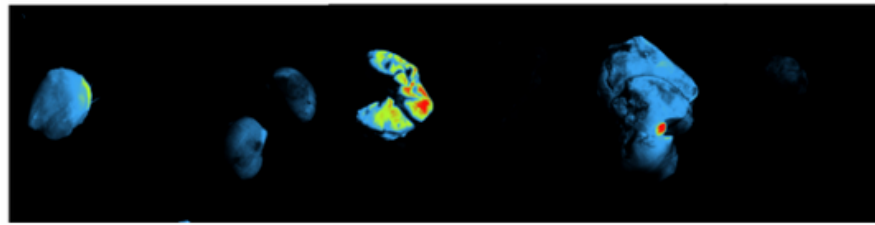

$6 \mathrm{~h}$

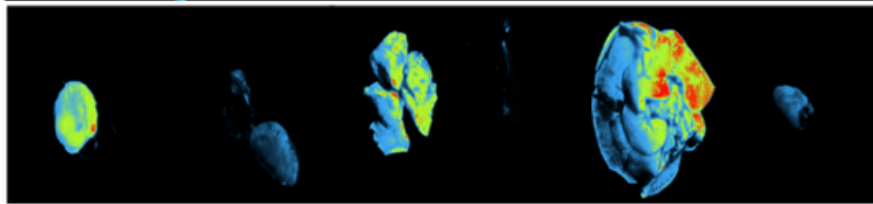

$24 \mathrm{~h}$

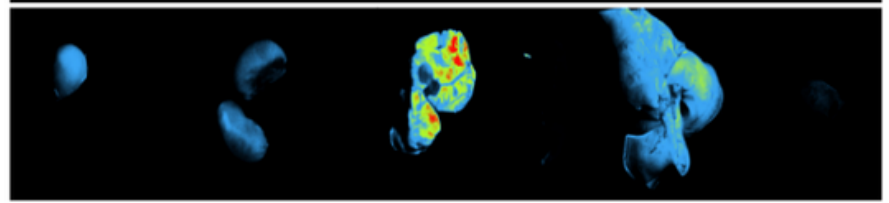

Figure 6

In Vivo Fluorescence Imaging. Fluorescence signal images of (A) tumor sites (C) the main organs and ex vitro tumor in tumor-bearing nude mice at different time. (B) Fluorescence signal quantitative diagram, (b) fluorescence signal diagram of EZAP with different concentrations excited at $365 \mathrm{~nm}$.

\section{Supplementary Files}


This is a list of supplementary files associated with this preprint. Click to download.

- Graphicalabstractimage.jpg

- schema1.png

- supportingInformation.docx 